\title{
Short-Term Power Load Forecasting Method Based on Improved Exponential Smoothing Grey Model
}

\author{
Jianwei Mi $\mathbb{D},{ }^{1,2}$ Libin Fan, ${ }^{1,2}$ Xuechao Duan $\mathbb{D}^{1,2}$ and Yuanying Qiu $\mathbb{D}^{1,2}$ \\ ${ }^{1}$ School of Mechano-Electronic Engineering, Xidian University, Xi'an 710071, China \\ ${ }^{2}$ Key Laboratory of Electronic Equipment Structure Design, Ministry of Education, Xidian University, \\ No. 2 South Taibai Road, Xian 710071, China \\ Correspondence should be addressed to Jianwei Mi; jwmi@xidian.edu.cn
}

Received 29 August 2017; Accepted 13 February 2018; Published 25 March 2018

Academic Editor: Emilio Turco

Copyright (C) 2018 Jianwei Mi et al. This is an open access article distributed under the Creative Commons Attribution License, which permits unrestricted use, distribution, and reproduction in any medium, provided the original work is properly cited.

\begin{abstract}
In order to improve the prediction accuracy, this paper proposes a short-term power load forecasting method based on the improved exponential smoothing grey model. It firstly determines the main factor affecting the power load using the grey correlation analysis. It then conducts power load forecasting using the improved multivariable grey model. The improved prediction model firstly carries out the smoothing processing of the original power load data using the first exponential smoothing method. Secondly, the grey prediction model with an optimized background value is established using the smoothed sequence which agrees with the exponential trend. Finally, the inverse exponential smoothing method is employed to restore the predicted value. The first exponential smoothing model uses the 0.618 method to search for the optimal smooth coefficient. The prediction model can take the effects of the influencing factors on the power load into consideration. The simulated results show that the proposed prediction algorithm has a satisfactory prediction effect and meets the requirements of short-term power load forecasting. This research not only further improves the accuracy and reliability of short-term power load forecasting but also extends the application scope of the grey prediction model and shortens the search interval.
\end{abstract}

\section{Introduction}

Short-term power load forecasting is a key issue for the operation and dispatch of power systems in order to prevent the serious consequences of flash and power failures. It is a prerequisite for the economic operation of power systems and the basis of dispatching and making startup-shutdown plans, which plays a key role in the automatic control of power systems [1-3]. Accurate power load forecasting not only helps users choose a more appropriate electricity consumption scheme and reduces a lot of electric cost expenditure while improving equipment utilization thus reducing the production cost and improving the economic benefit, but also is conducive to optimizing the resources of power systems, improving power supply capability and ultimately achieving the aim of energy conservation and emission reduction [46]. As the power system is increasingly complicated and the degree of electricity marketization is further enhanced, how to quickly and accurately predict short-term power loads has become one of the popular topics in the field of power load forecasting.

As a fundamental research, power load forecasting has been investigated for a long time. Many experts and scholars have done a lot of research on prediction theory and methods and put forward several prediction models and methods [711]. At present, the prediction method of power load can be divided into two categories [12-14]. One is the classical prediction method of statistical class, such as regression analysis, time series method, and grey prediction method. And the other is the novel prediction method of artificial intelligence class, such as expert systems and artificial neural networks. Because there are many factors affecting the shortterm power load and different prediction methods have different applications, none of these methods is applicable to all power systems, which need to choose different prediction models according to different power load conditions [15-18].

Grey system theory was proposed in 1982 [19]. It is a novel algorithm of coping with the problem of uncertainty 
with less data and poor information. Its essence is to estimate the development law of an object containing incomplete information based on the principle of grey system analysis $[20,21]$. Compared with other prediction methods, the grey prediction model has the characteristics of less data, high prediction precision, and no prior information. Therefore, it is suitable for short-term power load forecasting. China's power load has both the certainty increased year by year and the uncertainty affected by external factors, which agrees with the characteristics of "small sample, poor information" of the grey system, so it is rational to use the grey model for modeling prediction [22-24]. However, the $\operatorname{GM}(1,1)$ which is commonly used in the traditional grey prediction model is a biased exponential model. In particular, when the data fluctuates, its prediction error is too large to meet the requirements of the actual power load forecasting.

The traditional $\operatorname{GM}(1,1)$ model is only used for the modeling and prediction of single time series to reveal the inherent development law of the single variable. But the actual power system often contains multiple factor variables coupled with each other; that is, each factor variable in its development process is affected by other factors and also affects other factors at the same time. In order to get the predicted value that agrees with the actual situation, we should take the comprehensive influences of various factors on the predicted variables into consideration.

The traditional grey prediction model has many problems to be solved, such as its complex improved methods, the fact that it cannot comprehensively consider the effects of influencing factors, its limited application scope, and its prediction error failing to meet the requirement. Aimed at these problems, many scholars have proposed various improved methods $[25,26]$. Based on the analysis of these improved methods, this paper firstly employs the main influencing factor from various influencing factors using the grey correlation analysis. And then it establishes an improved exponential smoothing grey prediction model combining the exponential smoothing method and the characteristics of short-term power load, which carries out short-term load forecasting using the historical data of power load and influencing factors. The simulated results show that the method has a satisfactory prediction effect on the shortterm power load. The validity and feasibility of the prediction model are of great significance to solve the problem of the short-term power load forecasting in the development of smart grids in the future.

\section{The Exponential Smoothing Method and Traditional Grey Prediction Model}

2.1. The Exponential Smoothing Method. The exponential smoothing method is also a straightforward time series prediction method, which has the characteristics of simple calculation and convenient use. It is often applied to shortterm and ultrashort-term power load forecasting and has high precision [27]. The prediction for the linear model of the exponential smoothing method is shown in

$$
y_{t+p}=A_{t}+B_{t} \cdot p
$$

where $t$ is the current period, $p$ is the predicted period in advance, and $y_{t+p}$ is the predicted value in $t+p$ period. The parameters $A_{t}$ and $B_{t}$ are determined by

$$
\begin{aligned}
A_{t} & =2 S_{t}^{\prime}-S_{t}^{\prime \prime}, \\
B_{t} & =\frac{\alpha\left(S_{t}^{\prime}-S_{t}^{\prime \prime}\right)}{(1-\alpha)}, \\
S_{t}^{\prime} & =\alpha F_{t}+(1-\alpha) S_{t-1}^{\prime}, \\
S_{t}^{\prime \prime} & =\alpha S_{t}^{\prime}+(1-\alpha) S_{t-1}^{\prime \prime},
\end{aligned}
$$

where $\alpha$ is the smooth coefficient and $F_{t}$ is the original value at time $t$. $S_{t}^{\prime}$ and $S_{t-1}^{\prime}$ are the first smoothing values at time $t$ and time $t-1$, respectively. $S_{t}^{\prime \prime}$ and $S_{t-1}^{\prime \prime}$ are the second smoothing values at time $t$ and time $t-1$, respectively, as well as $S_{1}^{\prime}=S_{1}^{\prime \prime}=$ $F_{1}$, where $S_{1}^{\prime}$ and $S_{1}^{\prime \prime}$ represent the first smoothing value and the second smoothing value at the initial time, respectively, and $F_{1}$ represents the original value at the initial time.

From (2), we can know that the smooth coefficient $\alpha$ value directly affects the accuracy of the predicted value. Therefore, the most critical step in the exponential smoothing method is to determine the smooth coefficient. And it can help reduce the prediction error by finding out the optimal $\alpha$ value. The methods commonly used to determine the smooth coefficient are the empirical estimation method, trial and error, and others. However, the common drawback of the two methods is that forecasting researchers must perform the iterations and calculations several times to obtain an optimal $\alpha$ value which has a tight relationship with the knowledge, professional experience, and the number of calculations of the forecasting researchers. What is more, the forecasting process of this method (which is used to determine the smooth coefficient by the empirical estimation and trial-anderror methods) needs human intervention and thus has low automation and is an inefficient solving method. To overcome the drawback of the above two methods, the 0.618 method [28] can be used to search for the optimal smooth coefficient. However, the optimum result of the 0.618 method depends mainly on the objective function chosen.

2.2. The Traditional Grey Prediction Model. The grey prediction model is one of the core contents of the grey system theory. The most commonly used grey prediction model in power load forecasting is the $\operatorname{GM}(1,1)$ model, whose parameters indicate that the model establishes a first-order differential equation for one predicted variable to make predictions. As shown in Figure 1, the traditional grey prediction modeling process mainly includes accumulated generation, grey parameters calculation, solving the differential equation, and inverse accumulated generation. The detailed procedures can be found in [29]. The advantage of the traditional grey prediction model is that there is not much demand for the sample and it can get a better prediction effect in the case of few data samples. The disadvantage is that it can only make predictions for a single variable and requires that the data change be gentle and in accordance with the exponential 


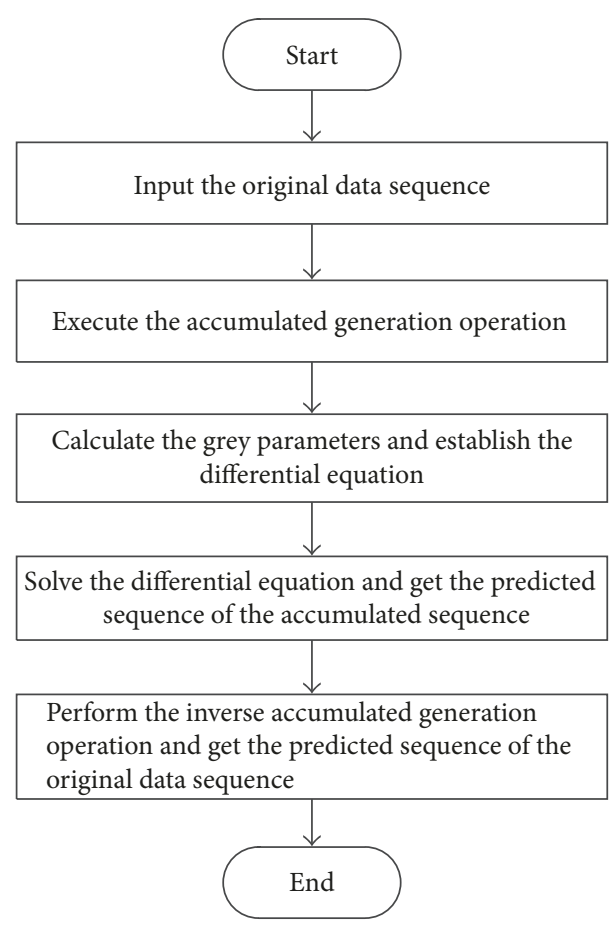

FIGURE 1: The flow diagram of the traditional grey prediction model.

change law; thus, the prediction effect is not satisfactory in case of data fluctuation.

\section{The Improved Exponential Smoothing Grey Model}

Because the traditional grey $\mathrm{GM}(1,1)$ prediction model is only applicable to the case in which the data change is relatively gentle, it can neither meet the actual forecasting requirements without an ideal prediction effect nor consider the effects of influencing factors on it for the case where the data sequence has a fast growth rate or large fluctuation. Aimed at the disadvantage of the $\operatorname{GM}(1,1)$ model (i.e., it cannot be applied to power load forecasting with fluctuation, complex environment, and obvious effects of influencing factors), an improved exponential smoothing grey prediction model is established in this paper using the selected main influencing factor variable and power load variable based on the analysis of short-term power load characteristics combining the grey correlation analysis, the exponential smoothing method, and the 0.618 method. By combining the influences of the main factors, the prediction model can expand the application scope of the grey prediction model, shorten the search interval when searching for the optimal smooth coefficient using the 0.618 method, and further improve the prediction accuracy and reliability.

As shown in Figure 2, the concrete processes of the improved exponential smoothing grey model are as follows.

Step 1. Input the real-time data of the original power load and perform the grey correlation analysis to determine the main influencing factor of the predicted object.
Step 2. Perform the first exponential smoothing processing to weaken its stochastic volatility and make it closer to the exponential trend.

Step 3. Make predictions for the smoothed sequence using the grey model with an optimized background value.

Step 4. The predicted results are restored to the predicted values of the original power load data and the data at the next prediction time through the inverse exponential smoothing processing.

Step 5. Judge whether the predicted results reach the requirement of the fitting error. If they do, then output the predicted results. If they do not, then the 0.618 method is introduced, which reselects the subinterval of smoothing coefficient and the pilot smoothing coefficient and then judges the pilot smoothing coefficient. If it reaches $\left|\alpha_{i}-\alpha_{i}^{\prime}\right|<\varepsilon$, where $\alpha_{i}$ and $\alpha_{i}^{\prime}$ are the first pilot smoothing coefficient and the second pilot smoothing coefficient in the $i$ th subinterval, respectively, and $\varepsilon$ is the accuracy requirement, then take the optimal smooth coefficient $\alpha_{i}^{*}=\left(\alpha_{i}+\alpha_{i}^{\prime}\right) / 2$ and continue the algorithm. If it does not, then take the $\alpha$ value with a smaller MAPE (Mean Absolute Percentage Error) value and continue the algorithm.

The key step in the above forecasting process is to dynamically update the original power load data. The total amount of the original power load data in the update process keeps unchanged, that is, selecting a suitable moving span. When moving a span every time, it removes the "oldest" data and adds the "latest" data so that each forecasting process 


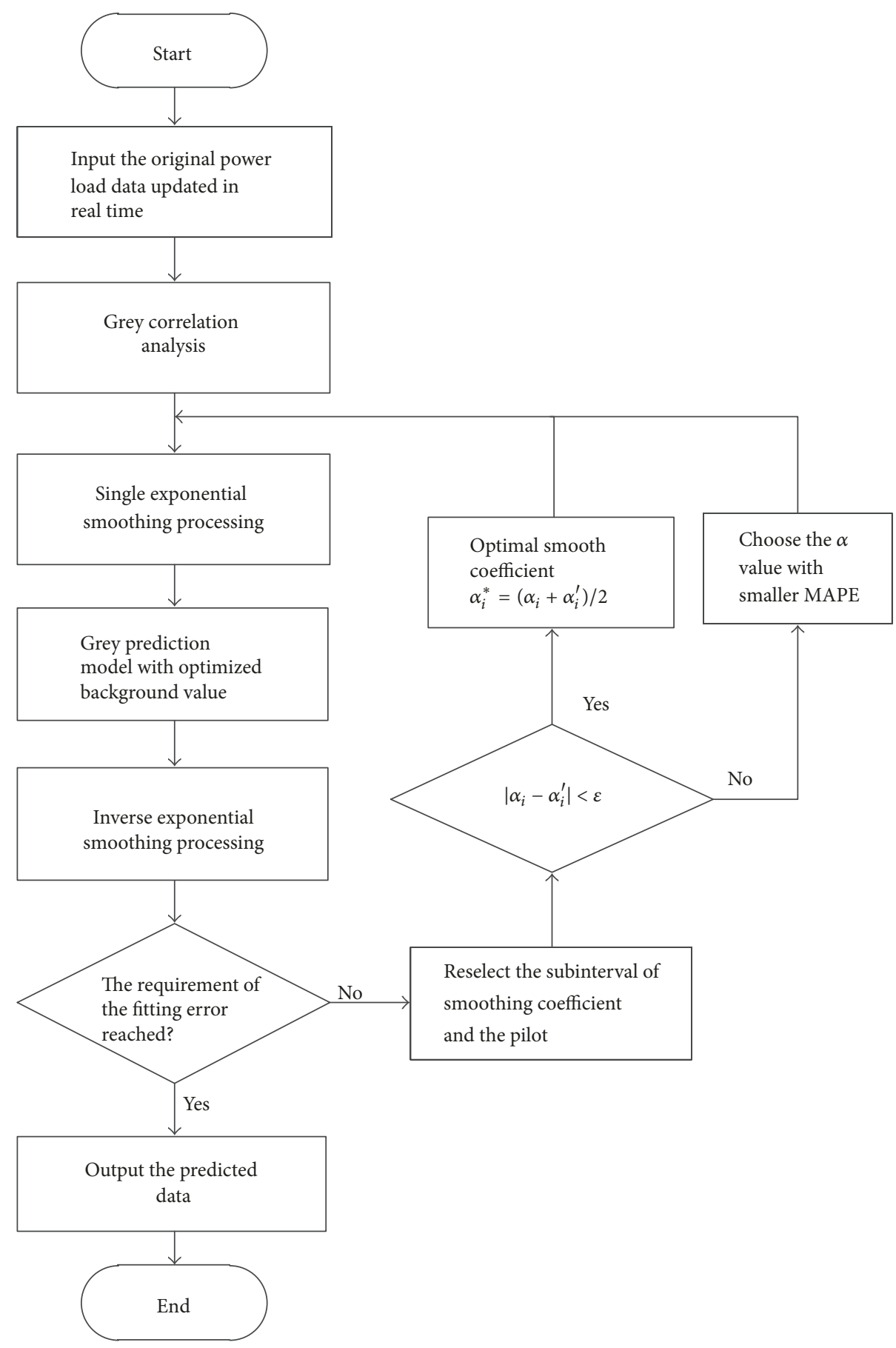

FIGURE 2: The flow diagram of the improved exponential smoothing grey model.

corresponds to a particular optimal smoothing coefficient, which can implement the real-time correction of the prediction model parameter when the memory occupation stays the same. In addition, the smoothing processing of the original data sequence is similar to that in [30], which can not only display the data more smoothly, but also eliminate the random errors to a certain degree.
3.1. The Grey Correlation Analysis. The grey correlation analysis is a multivariable statistical analysis method, whose basic idea is to judge whether there is a correlation between any two factor variables according to the similarity degree of the curves' geometrical shapes of various factor sequences. The closer the curve is, the closer the correlation between the corresponding sequences of variables is, that is, the greater 
the correlation degree is, and vice versa [31]. The specific steps are as follows:

(1) Determine the main behavior factor variable of the system $\mathbf{x}_{1}$ (the predicted object) and the influencing factor variable sequences $\mathbf{x}_{i}$ :

$$
\begin{aligned}
\mathbf{x}_{1} & =\left(x_{11}, x_{12}, \ldots, x_{1 k}\right), \\
\mathbf{x}_{i} & =\left(x_{i 1}, x_{i 2}, \ldots, x_{i k}\right), \quad(i=2,3, \ldots, n) .
\end{aligned}
$$

In the expression above, $k$ represents the data length of $\mathbf{x}_{1}$

(2) Normalize each variable sequence and get the initial values $\mathbf{x}_{i}^{\prime}$ as follows:

$$
\mathbf{x}_{i}^{\prime}=\frac{\mathbf{x}_{i}}{x_{i 1}}=\left(x_{i 1}^{\prime}, x_{i 2}^{\prime}, \ldots, x_{i k}^{\prime}\right), \quad(i=1,2, \ldots, n) .
$$

(3) Calculate the correlation coefficient between the main behavior sequence and each influencing factor sequence.

Let $\Delta_{i j}=\left|x_{1 j}^{\prime}-x_{i j}^{\prime}\right|(j=1,2, \ldots, k)$, where $x_{i j}^{\prime}$ represents the $j$ th initial value of the $i$ th variable. The difference sequences can be expressed as the following sequence: $\Delta_{\mathbf{i}}=$ $\left(\Delta_{i 1}, \Delta_{i 2}, \ldots, \Delta_{i k}\right)(i=2,3, \ldots, n)$. The maximum difference $M$ and the minimum difference $m$ can be calculated by the equations $M=\max _{i}\left\{\max _{j}\left\{\Delta_{i j}\right\}\right\}$ and $m=\min _{i}\left\{\min _{j}\left\{\Delta_{i j}\right\}\right.$. Therefore, the correlation coefficient can be calculated by the equation $\xi_{i j}=(m+\rho M) /\left(\Delta_{i j}+\rho M\right)$. In the equation, the parameter $\rho \in(0,1)$ and it is generally equal to 0.5 [32].

(4) Calculate the correlation degree.

Based on the correlation coefficients above, one can get the correlation degree $\gamma_{i}=(1 / k) \sum_{j=1}^{k} \xi_{i j},(i=2,3, \ldots, n)$, where $\gamma>0$. And then one can select the main influencing factor and eliminate the secondary factors according to the correlation degree.

By performing the grey correlation analysis for each influencing factor and the main behavior variable, the result can provide the basis for the selection of the variables in the prediction model and avoid that the unrelated factors or the factors with small correlation degree affect the prediction efficiency of the whole system and reduce the prediction accuracy.

3.2. The Improvements of the Traditional Grey Prediction Model. The traditional grey prediction model requires that the predicted load sequence should conform with the exponential trend. The improvement of smoothness of the load sequence helps to improve the prediction accuracy of the grey model. Therefore, the improvements of the traditional grey prediction model lie mainly in two aspects: (1) the transformation of the original sequence, that is, to improve its smoothness, which makes it closer to the exponential law, and (2) the optimization of model parameters, that is, to optimize and transform the background value used for solving the grey parameters. The original power load data is amended using the first exponential smoothing method of the exponential smoothing method in the improved grey prediction model, which weakens its stochastic volatility and makes the original data sequence more smoothly close to the exponential law. It also implements the prediction restoration of the original power load data and the data at the next prediction time using the inverse exponential smoothing in the final processing. As shown in Figure 3, the improved grey prediction model includes the following steps.

(1) Firstly, perform the grey correlation analysis for the input original data sequence $x_{i}^{0}$. And then perform the first exponential smoothing processing for the selected variables using (5) to improve the smoothness of the sequence and make it meet the requirement of the input data in the grey prediction model. The smooth coefficient in the first exponential smoothing equation is obtained by the optimization of the 0.618 method. Finally, perform the accumulated generation for the obtained smoothed sequence $S_{i}^{0}$. As shown in (6), we can obtain the accumulative sequence $y_{i}^{1}$, where the superscript 0 represents the sequence that did not undergo accumulated generation and the superscript 1 represents the sequence that underwent accumulated generation. Moreover, $i=1,2, \ldots, n, k=2,3, \ldots, q$, where $n$ represents the number of variables selected by the grey correlation analysis and $q$ represents the number of the original power load data:

$$
\begin{aligned}
& S_{i}^{0}(k)=\alpha^{*} x_{i}^{0}(k)+\left(1-\alpha^{*}\right) S_{i}^{0}(k-1), \\
& y_{i}^{1}(k)=S_{i}^{0}(k)+S_{i}^{0}(k+1) .
\end{aligned}
$$

(2) Optimize the background value $Z_{i}$ in the grey prediction model according to (7) using the optimal smooth coefficient $\alpha^{*}$ used in the first exponential smoothing processing. Calculate the data matrices $L$ and $R$ according to the optimized background value as shown in (8) and finally obtain the grey parameters $C$ and $D$ whose equations are as follows. The weight $\beta$ of the background value $Z_{i}$ in the grey prediction model is taken as the value related to the optimal smooth coefficient $\alpha^{*}$, which can avoid the error caused by fixing $\beta$ to 0.5 in the traditional grey prediction model and help to improve the prediction precision:

$$
\begin{aligned}
\beta & =\frac{1}{\alpha^{*}}-\frac{1}{e^{\alpha^{*}}-1}, \\
Z_{i}(k) & =\beta y_{i}^{1}(k-1)+(1-\beta) y_{i}^{1}(k) .
\end{aligned}
$$

In (8), the superscript 1 represents the notion that the sequence has been accumulated. And $i=1,2, \ldots, n, k=$ $2,3, \ldots, q$, where $n$ represents the number of variables selected by the grey correlation analysis and $q$ represents the number of the original power load data:

$$
L=\left[\begin{array}{ccccc}
Z_{1}(2) & Z_{2}(2) & \cdots & Z_{n}(2) & 1 \\
Z_{1}(3) & Z_{2}(3) & \cdots & Z_{n}(3) & 1 \\
\vdots & \vdots & \vdots & \vdots & \vdots \\
Z_{1}(q) & Z_{2}(q) & \cdots & Z_{n}(q) & 1
\end{array}\right] \text {, }
$$




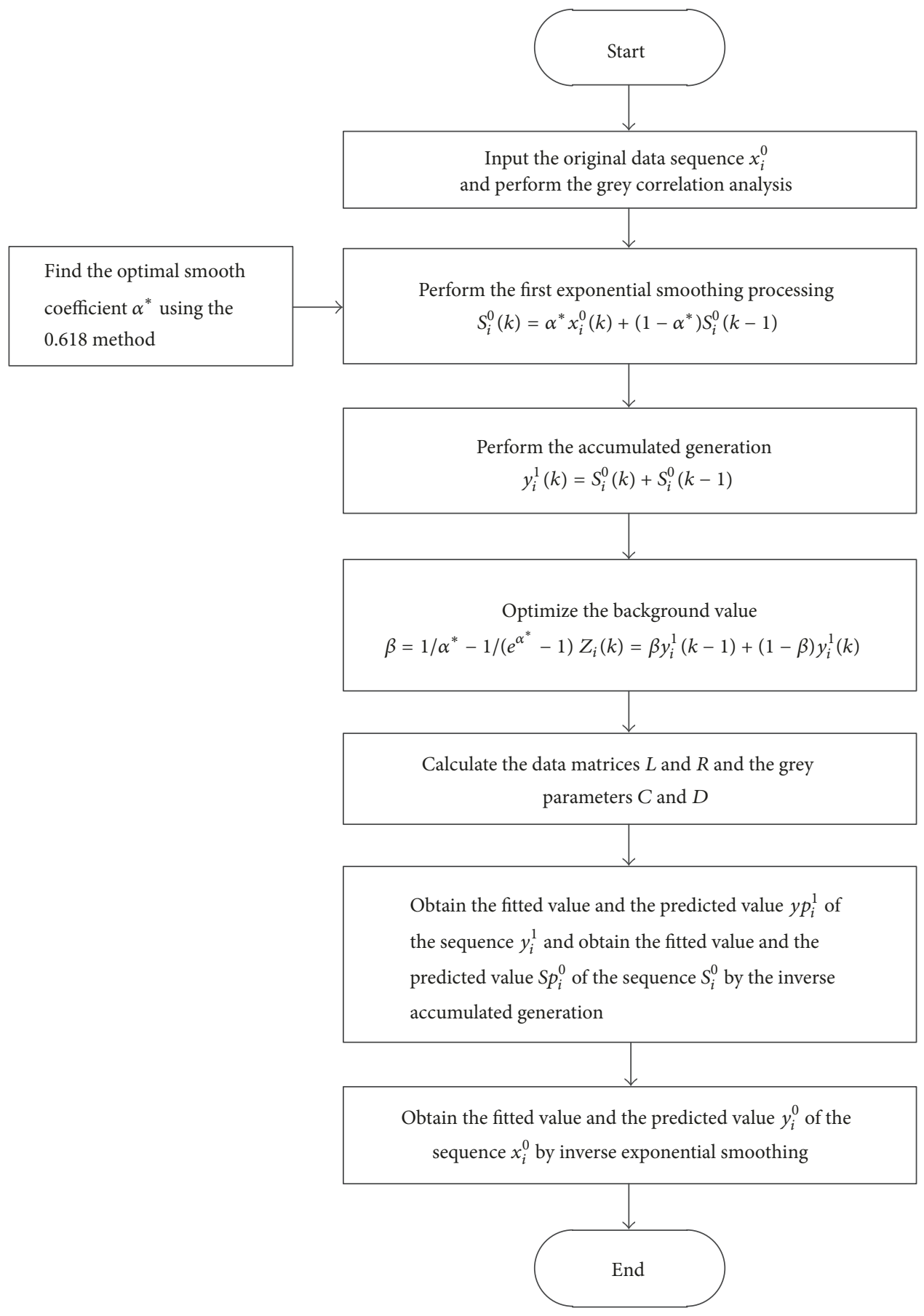

FIGURE 3: The structure diagram of the improved grey prediction model.

$$
\begin{aligned}
& R=\left[\begin{array}{cccc}
S_{1}^{0}(2) & S_{2}^{0}(2) & \ldots & S_{n}^{0}(2) \\
S_{1}^{0}(3) & S_{2}^{0}(3) & \cdots & S_{n}^{0}(3) \\
\vdots & \vdots & \vdots & \vdots \\
S_{1}^{0}(q) & S_{2}^{0}(q) & \cdots & S_{n}^{0}(q)
\end{array}\right] \\
& {\left[\begin{array}{c}
C^{T} \\
D^{T}
\end{array}\right]=H=\left(L^{T} L\right)^{-1} L^{T} R .}
\end{aligned}
$$

In (11), $H$ is a matrix with an order of $(n+1) \times n . C^{T}$ takes the first $n$ rows of the $H$ matrix and $D^{T}$ takes the last row of the $H$ matrix. To, respectively, transpose the two submatrices, one can get the grey parameters $C$ and $D$, where $C$ is a matrix with the order of $n \times n$ and $D$ is a matrix with the order of $n \times 1$.

(3) Obtain the predicted sequence $y p_{i}^{1}$ of the sequence $y_{i}^{1}$ according to the grey prediction (see (12)) and obtain the predicted sequence $S p_{i}^{0}$ of the sequence $S_{i}^{0}$ by the inverse accumulated generation (see (13)). 


$$
\begin{aligned}
& y p_{i}^{1}(k)=e^{C(k-1)}\left(y_{i}^{1}(1)+C^{-1} D\right)-C^{-1} D, \\
& S p_{i}^{0}(k)=y p_{i}^{1}(k)-y p_{i}^{1}(k-1) .
\end{aligned}
$$

(4) Put the predicted sequence $S p_{i}^{0}$ obtained by the grey prediction equation into the inverse exponential smoothing model as shown in (14) to realize the prediction restoration of the original power load data and the data at the next prediction time, and finally obtain the predicted sequence $y_{i}^{0}$ of the original power load data $x_{i}^{0}$ and the data at the next prediction time:

$$
y_{i}^{0}(k)=\frac{\left(S p_{i}^{0}(k)-\left(1-\alpha^{*}\right) S p_{i}^{0}(k-1)\right)}{\alpha^{*}} .
$$

3.3. The Improvement of the 0.618 Method. It can be seen from Figure 3 that the accuracy of the smooth coefficient is directly related to the prediction accuracy. Generally speaking, the MSE (Mean Square Error) or the MAD (Mean Absolute Deviation) will be chosen as the objective function in the 0.618 method to search for the optimal smooth coefficient. The value of MSE can be expressed as $f_{\text {MSE }}$ and its calculation formula is shown in (15). The value of MAD can be expressed as $f_{\text {MAD }}$ and its calculation formula is shown in (16). However, through the actual calculation, one can observe that choosing MAPE as the objective function can yield better effects. The value of MAPE can be expressed as $f_{\text {MAPE }}$ and its calculation formula is shown in (17).

$$
\begin{aligned}
f_{\mathrm{MSE}} & =\frac{\sum_{i=1}^{q}\left(x_{i}^{0}-y_{i}^{0}\right)^{2}}{q}, \\
f_{\mathrm{MAD}} & =\frac{\sum_{i=1}^{q}\left|x_{i}^{0}-y_{i}^{0}\right|}{q}, \\
f_{\mathrm{MAPE}} & =\frac{100}{q} \sum_{i=1}^{q}\left|\frac{x_{i}^{0}-y_{i}^{0}}{x_{i}^{0}}\right| .
\end{aligned}
$$

In the equations, $q$ is the number of the original power load data. $y_{i}^{0}$ is the predicted sequence of $x_{i}^{0}$, which is related to the smooth coefficient. One can see that $f_{\text {MAPE }}$ actually is a function of smooth coefficient. One can observe from (15) that MSE is the average of the sums of squares of the errors between the actual value and the predicted value, which makes MSE unable to measure the unbiasedness. Similarly, one can observe from (16) that MAD is the average of the sums of the absolute deviation between the actual value and the predicted value, but MAD fails to reflect the effect of the deviation on the actual value. Therefore, compared with MSE and MAD, it can not only more accurately reflect the deviation between the predicted value and the actual value, but also effectively measure the unbiasedness and improve the reliability of prediction using MAPE as the objective function of the 0.618 method.

The specific processes of the improved 0.618 method can be divided into five steps, as shown in Figure 4.

(1) Let $\varepsilon=0.01$ and divide the smooth coefficient $\alpha \in$ $(0,1)$ into 10 equidistant subintervals: $\alpha_{1} \in(0,0.1), \alpha_{2} \in$
$(0.1,0.2), \ldots, \alpha_{10} \in(0.9,1)$. Select a subinterval $\alpha_{i} \in\left(a_{0}, b_{0}\right)$ ( $i=1,2, \ldots, 10)$, where $a_{0}$ and $b_{0}$ represent the left and right endpoints of the $i$ th subinterval, respectively; proceed to the next step. Furthermore, the smooth coefficient interval is divided into 10 equidistant subintervals for meeting the requirement that the objective function of the 0.618 method is a unimodal function. Because it is difficult to prove that MAPE is a unimodal function in the whole interval of $[0,1]$, it is possible to distribute the extreme points of MAPE in different subintervals by dividing the interval equally so that MAPE is a unimodal function in each subinterval. Even though MAPE is not a unimodal function in each subinterval, there is a little impact on searching for the optimal value. The reason is that the function value at every point in each subinterval is close to its minimum and it is sufficient to meet the precision requirement of $\varepsilon=0.01$ when the interval length is small to a certain extent (equal to 0.1 here). The basic idea of the 0.618 method can be found in [33].

(2) Take the first trial point and let the first pilot smoothing coefficient $\alpha_{i}=0.618 a_{0}+0.382 b_{0}$.

(3) Take the second trial point and let the second pilot smoothing coefficient $\alpha_{i}^{\prime}=0.382 a_{0}+0.618 b_{0}$.

(4) Judge whether $\left|\alpha_{i}-\alpha_{i}^{\prime}\right|<\varepsilon$ holds or not. If it holds, then take the optimal smooth coefficient $\alpha_{i}^{*}=\left(\alpha_{i}+\alpha_{i}^{\prime}\right) / 2$ and proceed to the subsequent process. If it does not, then proceed to step (5).

(5) Calculate the MAPE values $f_{1}\left(\alpha_{i}\right)$ and $f_{2}\left(\alpha_{i}^{\prime}\right)$ corresponding to the two trial points and compare their values. If $f_{1}\left(\alpha_{i}\right)<f_{2}\left(\alpha_{i}^{\prime}\right), a_{0}$ keeps constant, $b_{0}=\alpha_{i}^{\prime}, \alpha_{i}^{\prime}=\alpha_{i}$, and $f_{2}\left(\alpha_{i}^{\prime}\right)=f_{1}\left(\alpha_{i}\right)$, and proceed to step (2). If $f_{1}\left(\alpha_{i}\right)>f_{2}\left(\alpha_{i}^{\prime}\right)$, $b_{0}$ keeps constant, $a_{0}=\alpha_{i}, \alpha_{i}=\alpha_{i}^{\prime}$, and $f_{1}\left(\alpha_{i}\right)=f_{2}\left(\alpha_{i}^{\prime}\right)$, and proceed to step (3).

\section{Simulated Results and Discussion}

As shown in Table 1, the electricity consumption of the whole society and the national economic indicators, that is, the influencing factors data in 8 time periods of Fujian Province in [32], are adopted. The factors affecting the electricity consumption $x_{1}$ in the table include the GDP (Gross Domestic Product) $x_{2}$, the total population $x_{3}$, and the import and export total $x_{4}$. In order to verify the validity of the proposed improved prediction algorithm, this paper realized the power load forecasting algorithm based on the improved exponential smoothing grey model, which builds the model using the electricity consumption data and its influencing factors data in 8 time periods shown in Table 1 and predicts the electricity consumption data in the next 2 time periods.

(1) Determine the main factor variable affecting the power load forecasting using the grey correlation analysis.

One can calculate the correlation degree of 3 influencing factors in Table 1 for the power load, and the results are shown in Table 2.

One can observe from the calculation results of the correlation degree in Table 2 that the correlation degree between the import and export total and the electricity consumption is the largest one; that is, the influencing factor 


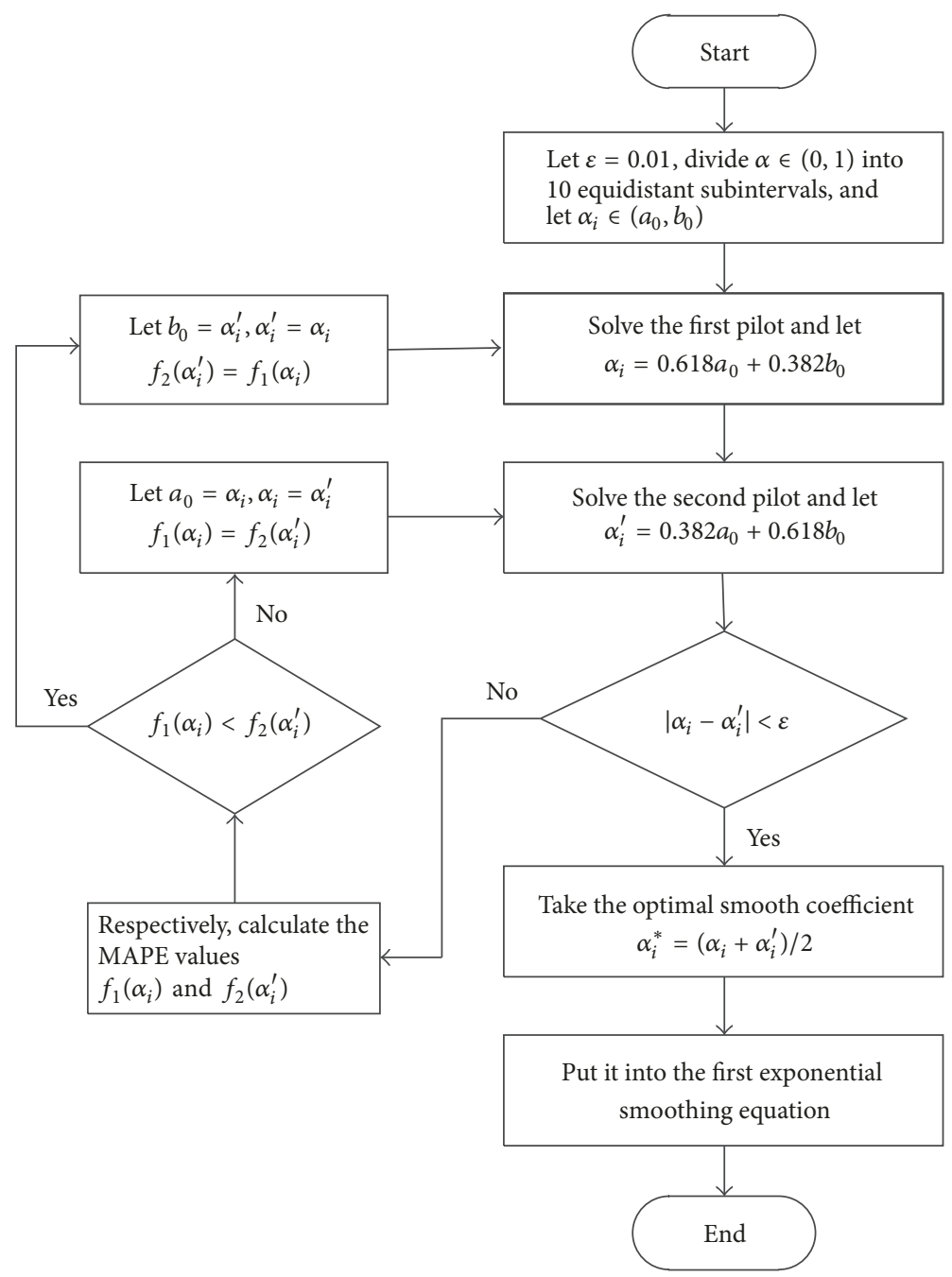

FIGURE 4: The structure diagram of the improved 0.618 method.

TABLE 1: The original electricity consumption and its influencing factors data.

\begin{tabular}{lcccc}
\hline Time $/ t$ & $x_{1}(\mathrm{GWh})$ & $x_{2}($ billion $\$)$ & $x_{3}($ million $\$)$ & $x_{4}(\mathrm{million} \$)$ \\
\hline 1 & 18701.75 & 18.805 & 31.50 & 10041.81 \\
2 & 22364.49 & 27.928 & 31.83 & 12189.53 \\
3 & 25947.70 & 35.765 & 32.37 & 14445.69 \\
4 & 27994.90 & 42.668 & 32.61 & 15519.72 \\
5 & 29973.13 & 49.575 & 32.82 & 17952.80 \\
6 & 32019.54 & 54.776 & 32.99 & 17160.65 \\
7 & 35337.03 & 59.171 & 33.16 & 17619.56 \\
8 & 40151.49 & 65.335 & 34.10 & 21223.32 \\
\hline
\end{tabular}

"the import and export total" has the greatest influence on the electricity consumption. Therefore, the import and export total is chosen as the main influencing factor variable.

(2) Build the improved exponential smoothing grey model and make predictions.

After determining the main factor variable according to Table 2, one can build the multivariable grey model of the electricity consumption and the import and export total using the original data in Table 1 . The grey parameters can be obtained by (11). Subsequently, the corresponding differential equations are solved. The data in the first 8 time periods are used to fit the model and the fitted results are shown in Table 3. The fitted errors in Table 3 are calculated by (17), such as $(|22364.49-23037.539| / 22364.49) \times 100 \%=$ $3.01 \%$. The data in the last 2 time periods are used to verify the performance of the prediction model and the 
TABLE 2: The correlation degree between each influencing factor and electricity consumption.

\begin{tabular}{lccc}
\hline Influencing factors & $\mathrm{GDP} / x_{2}$ & Total population $/ x_{3}$ & Import and export total $/ x_{4}$ \\
\hline Correlation degree & 0.5185 & 0.6110 & 0.9243 \\
\hline
\end{tabular}

TABLE 3: Comparison of the actual values and the fitted values of electricity consumption.

\begin{tabular}{|c|c|c|c|c|c|}
\hline \multirow{2}{*}{ Time/t } & \multirow{2}{*}{ Original data } & \multicolumn{2}{|c|}{ The improved model } & \multicolumn{2}{|c|}{ The model in [32] } \\
\hline & & Fitted data & Error/\% & Fitted data & Error $/ \%$ \\
\hline 1 & 18701.75 & 18701.75 & 0 & 18701.75 & 0 \\
\hline 2 & 22364.49 & 23037.539 & 3.01 & 22891.98 & 2.36 \\
\hline 3 & 25947.70 & 24884.68 & 4.1 & 24724.49 & 4.71 \\
\hline 4 & 27994.90 & 26963.009 & 3.69 & 26747.38 & 4.46 \\
\hline 5 & 29973.13 & 29326.489 & 2.16 & 28999.09 & 3.25 \\
\hline 6 & 32019.54 & 32046.51 & 0.08 & 31531.90 & 1.52 \\
\hline 7 & 35337.03 & 35218.013 & 0.34 & 34418.07 & 2.60 \\
\hline 8 & 40151.49 & 38967.811 & 2.95 & 41695.84 & 3.85 \\
\hline
\end{tabular}

TABLE 4: Comparison of the actual values and the predicted values of electricity consumption.

\begin{tabular}{|c|c|c|c|c|c|c|c|c|c|}
\hline \multirow{2}{*}{ Time } & \multirow{2}{*}{ Original values } & \multicolumn{2}{|c|}{ The improved model } & \multicolumn{2}{|c|}{$\operatorname{GM}(1,1)$ model } & \multicolumn{2}{|c|}{$\operatorname{GM}(1,4)$ model } & \multicolumn{2}{|c|}{ The model in [32] } \\
\hline & & Predicted values & Error $/ \%$ & Predicted values & Error/\% & Predicted values & Error $/ \%$ & Predicted values & Error $/ \%$ \\
\hline 9 & 43918.60 & 43465.89 & 1.03 & 43017.38 & 2.05 & 42963.27 & 2.18 & 43465.89 & 1.03 \\
\hline 10 & 49682.87 & 48940.73 & 1.49 & 47065.22 & 5.27 & 48044.23 & 3.30 & 48907.30 & 1.56 \\
\hline $\mathrm{Ave}$ & erage error \% & 1.26 & & 3.66 & & 2.74 & & 1.30 & \\
\hline
\end{tabular}

predicted results are shown in Table 4 . The predicted errors in Table 4 are calculated by (17), such as (|43918.60 $43465.89 \mid / 43918.60) \times 100 \%=1.03 \%$.

It can be seen from Table 3 that the fitted effect obtained by the improved prediction model is better than that in [32]. The parameters of the improved prediction model can be modified and optimized in real time according to the trend of the actual data. However, because the parameters of the model in [32] are fixed and the original data is not processed by any smoothing operations, the trend of the fitted data will be somewhat deviated from the trend of the actual data.

It can be seen from Table 4 that the predicted effect obtained by the improved prediction model is the best and its predicted average MAPE value is $1.26 \%$ which meets the requirement that the average error in short-term power load forecasting should be around 3\%. Although the error of the improved prediction model is only slightly smaller than that in [32], the electricity consumption difference will be large when used in an actual application, for example, $50000 \times 0.0126=630(\mathrm{GWh}), 50000 \times 0.013=650$ $(\mathrm{GWh})$, and the electricity consumption difference $\mathrm{EC}_{d}=20$ (GWh). If such a large electricity consumption difference is taken into account, it will save a very large economic cost. Especially for the large industrial consumers with a large electricity consumption base, it will be conducive to selecting a more reasonable charging mode by accurately forecasting the power demand for the next month, which makes the economic effect more obvious. This phenomenon also illustrates three problems: (1) if the influencing factors were not taken into consideration in the prediction model, it would lead to a poor prediction accuracy, such as the $\operatorname{GM}(1,1)$ model; (2) if other secondary influencing factors besides the main influencing factor were taken into consideration in the prediction model, the prediction error would also increase, such as the $\operatorname{GM}(1,4)$ model; (3) if the influencing factors were considered in the prediction model but the model parameters were not optimized, it would also lead to an increase in prediction error, such as the model adopted in [32]. In order to show the degree of deviation between the actual value and the predicted value more intuitively, the curves of the actual value and the predicted value are plotted in Figure 5.

It can be seen from Figure 5 that the trend of predicted values of power load is very close to the trend of actual value and the overall predicted effect is satisfactory. The simulated results show that the improved exponential smoothing grey prediction model is feasible and effective for short-term power load forecasting, which improves the prediction accuracy of the prediction model. Moreover, the introduction of the 0.618 method improves the solution efficiency and the automation and makes the predicted results highly reliable, which basically overcomes the shortcomings of the traditional grey prediction algorithm. Besides, the algorithm is straightforward to be realized.

\section{Conclusion}

The traditional grey prediction model has found wide applications in the field of power load forecasting because of its characteristics of simple principle, lower sample data 


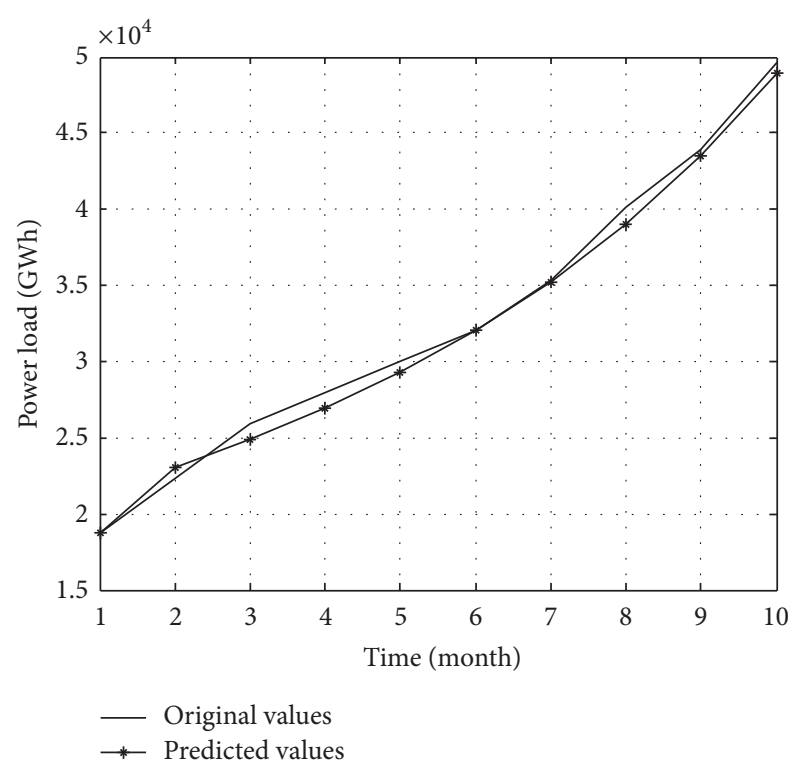

Figure 5: Comparison curves of the original values and the predicted values.

requirement, and ability to tackle uncertain problems. However, the disadvantages of the model itself result in its defective prediction effect and thus inability to meet the actual forecasting requirement. Aiming at the shortcomings of the above prediction model, this paper proposed a shortterm power load forecasting method based on the improved exponential smoothing grey model which not only preserves the advantages of the traditional grey prediction model for dealing with the poor information, but also analyzes the various influencing factors affecting power load forecasting using the grey correlation analysis and determines the main influencing factor. The improved prediction model reduces the calculation quantity and improves the prediction efficiency because it does not consider too much the secondary factors which can reduce the prediction efficiency. Some conclusions can be drawn as follows:

(1) The first exponential smoothing model is employed to deal with the original power load data in the improved prediction model, which not only weakens the randomness but also improves the smoothness of data. The smoothing processing makes it close to the exponential trend, which meets the requirement of the input data in the grey prediction model and contributes to further improving the prediction accuracy.

(2) The 0.618 method is introduced in the first exponential smoothing process and MAPE is chosen as the objective function to search for the optimal smooth coefficient, which enhances the reliability of prediction.

(3) The background value of the traditional grey prediction model is also optimized, which can implement the real-time correction of the prediction model parameter.

\section{Conflicts of Interest}

The authors declare that there are no conflicts of interest regarding the publication of this paper.

\section{Acknowledgments}

This work is partially supported by the 111 Project B14042 and the National Natural Science Foundation of China under Grants nos. 51490660 and 51405362.

\section{References}

[1] Y. He, Q. Xu, J. Wan, and S. Yang, "Short-term power load probability density forecasting based on quantile regression neural network and triangle kernel function," Energy, vol. 114, pp. 498-512, 2016.

[2] Z. Zhang and W. Gong, "Short-term load forecasting model based on quantum elman neural networks," Mathematical Problems in Engineering, vol. 2016, Article ID 7910971, 2016.

[3] X. Wang and Y. Wang, "A Hybrid Model of EMD and PSOSVR for Short-Term Load Forecasting in Residential Quarters," Mathematical Problems in Engineering, vol. 2016, Article ID 9895639, 2016.

[4] R. Hu, S. Wen, Z. Zeng, and T. Huang, "A short-term power load forecasting model based on the generalized regression neural network with decreasing step fruit fly optimization algorithm," Neurocomputing, vol. 221, pp. 24-31, 2017.

[5] H. Li, L. Cui, and S. Guo, "A hybrid short-term power load forecasting model based on the singular spectrum analysis and autoregressive model," Advances in Electrical Engineering, vol. 2014, Article ID 424781, 7 pages, 2014.

[6] H. Yuansheng, H. Shenhai, and S. Jiayin, "A novel hybrid method for short-term power load forecasting," Journal of Electrical and Computer Engineering, vol. 2016, Article ID 2165324, 2016.

[7] Y. He, R. Liu, H. Li, S. Wang, and X. Lu, "Short-term power load probability density forecasting method using kernel-based support vector quantile regression and Copula theory," Applied Energy, vol. 185, pp. 254-266, 2017.

[8] M. Capuno, J.-S. Kim, and H. Song, "Very short-term load forecasting using hybrid algebraic prediction and support vector regression," Mathematical Problems in Engineering, vol. 2017, Article ID 8298531, 2017.

[9] H. J. Sadaei, F. G. Guimaraes, C. Silva, M. H. Lee, and T. Eslami, "Short-term load forecasting method based on fuzzy time series, seasonality and long memory process," International Journal of Approximate Reasoning, vol. 83, pp. 196-217, 2017.

[10] A. Laouafi, M. Mordjaoui, S. Haddad, T. E. Boukelia, and A. Ganouche, "Online electricity demand forecasting based on an effective forecast combination methodology," Electric Power Systems Research, vol. 148, pp. 35-47, 2017.

[11] Y. Chen, P. Xu, Y. Chu et al., "Short-term electrical load forecasting using the Support Vector Regression (SVR) model to calculate the demand response baseline for office buildings," Applied Energy, vol. 195, pp. 659-670, 2017.

[12] B. Wang, D. Wang, and S. Zhang, "Short-term Distributed Power Load Forecasting Algorithm Based on Spark and IPPSO_LSSVM," Electric Power Automation Equipment, vol. 36, no. 1, pp. 117-122, 2016. 
[13] S. Brodowski, A. Bielecki, and M. Filocha, "A hybrid system for forecasting 24-h power load profile for Polish electric grid," Applied Soft Computing, vol. 58, pp. 527-539, 2017.

[14] M. B. Germi, M. Mirjavadi, A. S. S. Namin, and A. Baziar, "A hybrid model for daily peak load power forecasting based on SAMBA and neural network," Journal of Intelligent \& Fuzzy Systems: Applications in Engineering and Technology, vol. 27, no. 2, pp. 913-920, 2014.

[15] L. Xiao, W. Shao, C. Wang, K. Zhang, and H. Lu, "Research and application of a hybrid model based on multi-objective optimization for electrical load forecasting," Applied Energy, vol. 180, pp. 213-233, 2016.

[16] S. Zhang, R. Shi, L. Zhang, B. Yan, H. Zhang, and P. He, "Improvement of chaotic forecasting model and its application in power daily load forecasting," Chinese Journal of Scientific Instrument, vol. 37, no. 1, pp. 208-214, 2016.

[17] L. Xiao, W. Shao, T. Liang, and C. Wang, "A combined model based on multiple seasonal patterns and modified firefly algorithm for electrical load forecasting," Applied Energy, vol. 167, pp. 135-153, 2016.

[18] K. G. Boroojeni, M. H. Amini, S. Bahrami, S. S. Iyengar, A. I. Sarwat, and O. Karabasoglu, "A novel multi-time-scale modeling for electric power demand forecasting: From short-term to medium-term horizon," Electric Power Systems Research, vol. 142, pp. 58-73, 2017.

[19] N. Xie and S. Liu, "Interval grey number sequence prediction by using non-homogenous exponential discrete grey forecasting model," Journal of Systems Engineering and Electronics, vol. 26, no. 1, pp. 96-102, 2015.

[20] N. Xu, Y. Dang, and Y. Gong, "Novel grey prediction model with nonlinear optimized time response method for forecasting of electricity consumption in China," Energy, vol. 118, pp. 473-480, 2017.

[21] L. Jian-Kai, C. Cattani, and S. Wan-Qing, "Power load prediction based on fractal theory," Advances in Mathematical Physics, vol. 2015, Article ID 827238, 2015.

[22] B. Zeng and C. Li, "Forecasting the natural gas demand in China using a self-adapting intelligent grey model," Energy, vol. 112, pp. 810-825, 2016.

[23] S. Liu and L.-X. Tian, "The study of long-term electricity load forecasting based on improved grey prediction model," in Proceedings of the 12th International Conference on Machine Learning and Cybernetics, ICMLC 2013, pp. 653-657, China, July 2013.

[24] J. Massana, C. Pous, L. Burgas, J. Melendez, and J. Colomer, "Short-term load forecasting for non-residential buildings contrasting artificial occupancy attributes," Energy and Buildings, vol. 130, pp. 519-531, 2016.

[25] H. Zhao and S. Guo, "An optimized grey model for annual power load forecasting," Energy, vol. 107, pp. 272-286, 2016.

[26] M. Y. Tong, X. H. Zhou, and B. Zeng, “The background value optimization method in grey NGM $(1,1, \mathrm{k})$ model," Control and Decision, vol. 32, no. 3, 2017.

[27] P. Ji, D. Xiong, P. Wang, and J. Chen, "A study on exponential smoothing model for load forecasting," in Proceedings of the 2012 Asia-Pacific Power and Energy Engineering Conference, APPEEC 2012, China, March 2012.

[28] J. A. Koupaei, S. Hosseini, and F. M. Ghaini, "A new optimization algorithm based on chaotic maps and golden section search method," Engineering Applications of Artificial Intelligence, vol. 50, pp. 201-214, 2016.
[29] Y. Yang and D. Xue, "Continuous fractional-order grey model and electricity prediction research based on the observation error feedback," Energy, vol. 115, pp. 722-733, 2016.

[30] E. Cadenas, O. A. Jaramillo, and W. Rivera, "Analysis and forecasting of wind velocity in chetumal, quintana roo, using the single exponential smoothing method," Journal of Renewable Energy, vol. 35, no. 5, pp. 925-930, 2010.

[31] X. Xia, Y. Sun, K. Wu, and Q. Jiang, "Optimization of a straw ring-die briquetting process combined analytic hierarchy process and grey correlation analysis method," Fuel Processing Technology, vol. 152, pp. 303-309, 2016.

[32] Y. P. Wang, D. X. Huang, H. Q. Xiong, and Y. L. Niu, "Using relational analysis and multi-variable grey model for electricity demand forecasting in smart grid environment," Power System Protection and Control, vol. 40, no. 1, pp. 96-100, 2012.

[33] Y. X. Yuan and W. Y. Sun, Optimization theory and method, Science Press, Beijing, China, 1997, 96-99. 


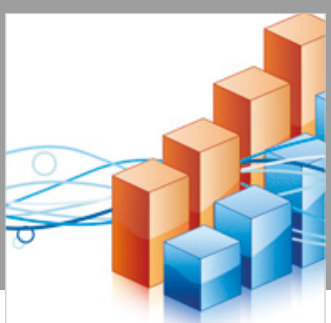

Advances in

Operations Research

\section{-n-m}
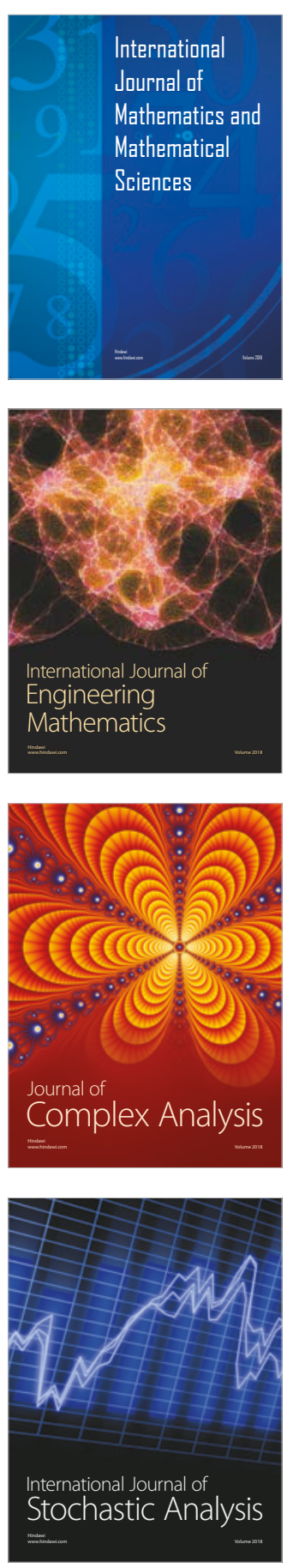
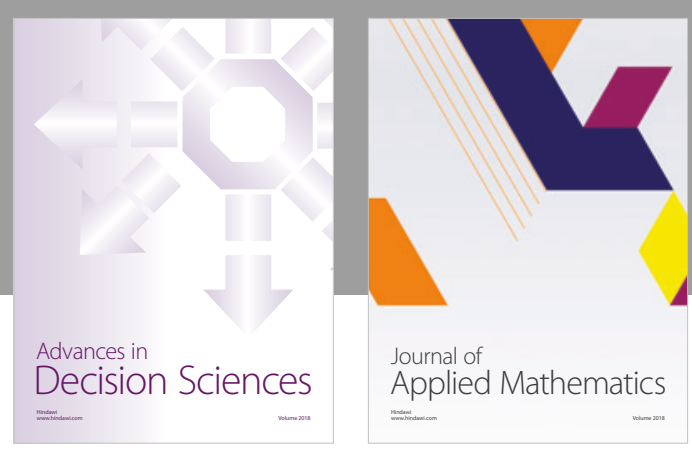

Journal of

Applied Mathematics
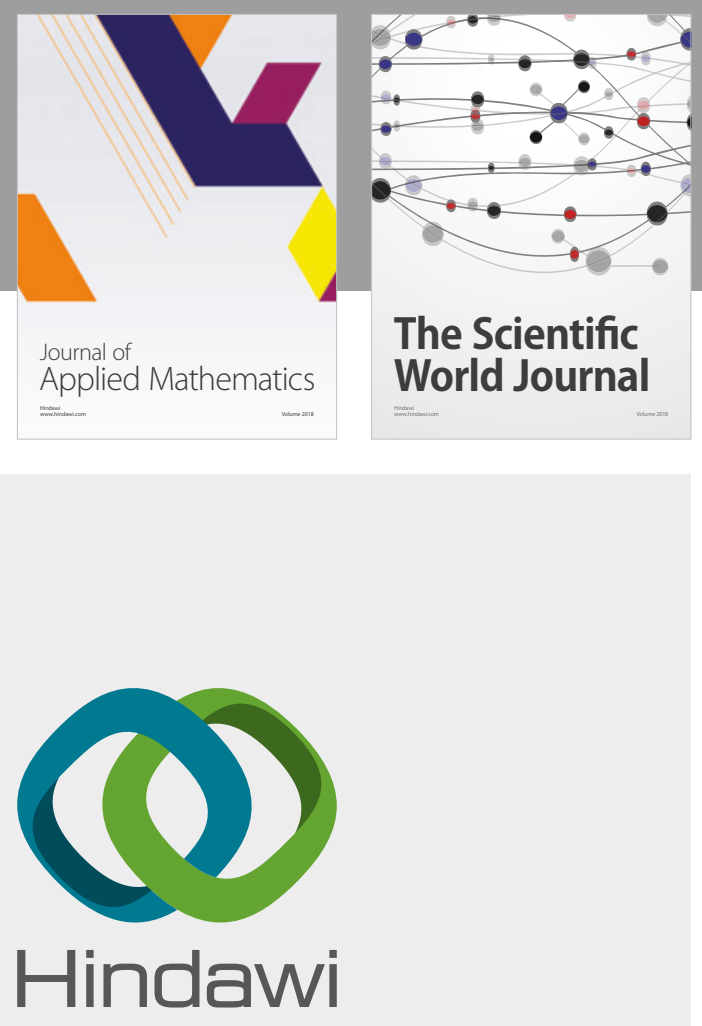

Submit your manuscripts at

www.hindawi.com

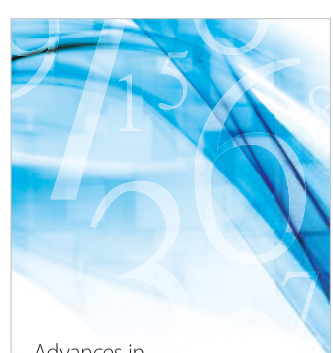

Advances in
Numerical Analysis
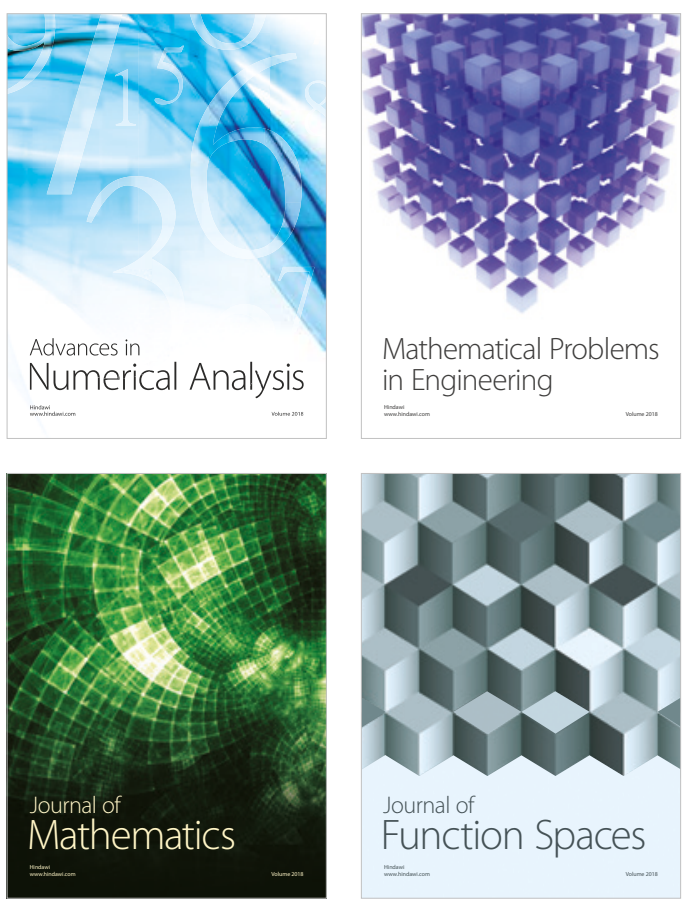

Mathematical Problems in Engineering

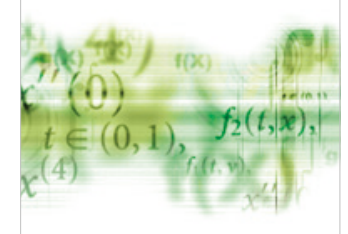

International Journal of

Differential Equations

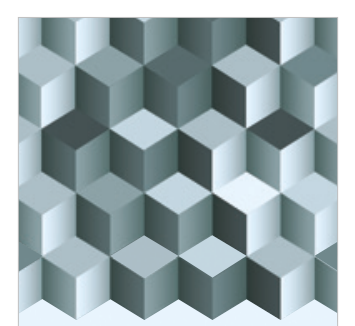

Journal of

Function Spaces

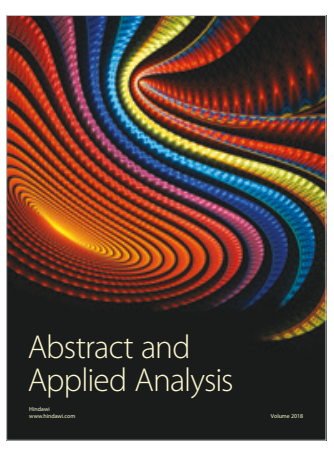

The Scientific

World Journal

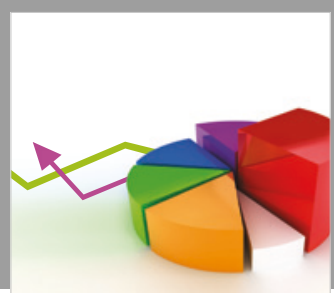

Journal of

Probability and Statistics
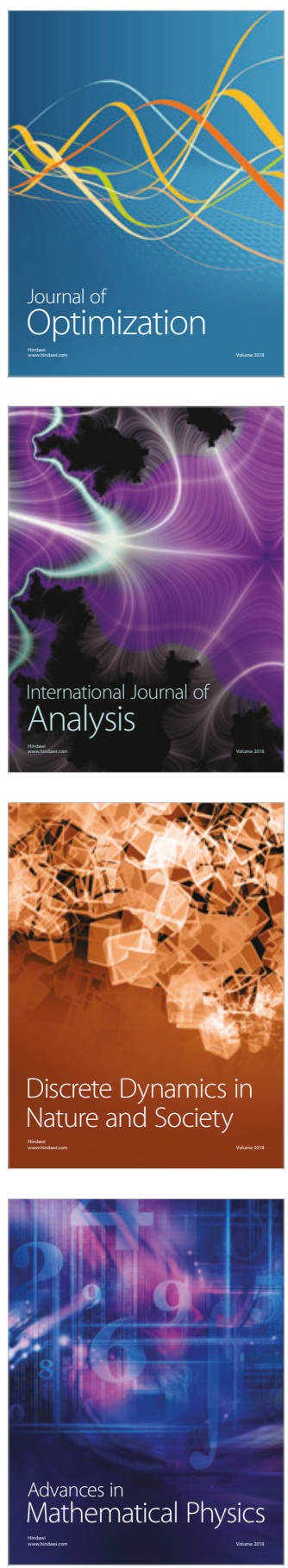